\title{
LA GESTIÓN DEL CONOCIMIENTO EN EL SIGLO XXI
}

\section{Augusto Bernuy Alva}

\section{Resumen}

En el mundo existe un desarrollo espectacular en la gestión del conocimiento, por ejemplo las conferencias IC3K; además, el prestigioso Massachusetts Institute of Technology cuenta con el MIT Center for Collective Intelligence para conducir investigaciones sobre como las TIC están cambiando la forma de trabajar de las personas, acerca de la eficiencia organizacional, la productividad, equipos de trabajo y liderazgo. Entonces, gestionar el conocimiento lleva a establecer condiciones para que la organización aprenda, con diferentes enfoques, desde formas de poner a disposición la información hasta sistemas que permitan solucionar problemas complejos, por ello se buscan crear nuevas formas de utilizar los recursos y lograr el desarrollo de nuevos sistemas alineados a nuevos procesos que al final permitirán mejorar la productividad, eficiencia y eficacia de la organización obteniendo ventaja competitiva sostenible. Este artículo presenta los modelos y estrategias que permitan utilizar las tendencias tecnológicas con la finalidad de desarrollar nuevos sistemas que mejoren la productividad yeficiencia organizacional.

Palabras clave: gestión del conocimiento, uso de tecnologías avanzadas, toma de decisiones organizacionales

\section{Knowledge management in the twenty-first century}

\section{Summary}

In the world, there is a spectacular development in the knowledge management, for example the conferences IC3K; in addition, the prestigious Massachusetts Institute of Technology relies on the MIT Center for Collective Intelligence to conduct research on how ICTS are changing the way people work; among others; then, an adequate knowledge management leads to create the conditions for the organization to learn, with different approaches, from the provision of timely information to systems that allow to solve complex problems; it is for this reason that they seek to create new ways to use resources and to achieve the development of systems aligned to new processes that allow us to improve the productivity, efficiency and effectiveness of the organizations, gaining a sustainable competitive advantage. This article presents the models and strategies that allow you to use the technology trends to develop new systems that improve the productivity and organizational efficiency.

Key words: knowledge management, use of advanced technologies, organizational decision making 


\section{Evolución de la gestión de la información}

La caída del valor de las acciones de Apple, primero en enero y luego en octubre del 2011, luego de los anuncios del mal estado de salud (Michán, 2011) y del fallecimiento de su cofundador Steve Jobs (Reuters, 2011), es una muestra de la importancia que tiene el conocimiento y el talento de las personas en la sociedad de la información, y la nueva economía basada en el conocimiento. Una empresa tiene muchas y variadas necesidades que van desde lo operativo a lo estratégico; desde una capacidad de almacenar y recuperar información, pasando por la imperiosa necesidad de identificar el valor y la experiencia de su capital humano, de modo que estén disponibles cuando las personas no estén presentes; por ejemplo, como le ocurrió a Apple con la enigmática dependencia hacia su fundador, o con alguna persona que luego de acumular 15 años de experiencia en cualquier empresa del mundo ha desarrollado las habilidades de toma de decisiones en temas operativos, tema de gestión y temas estratégicos, y literalmente se hace indispensable.

En primer lugar, las decisiones son variadas, por ello las necesidades de conocimiento organizacional varían dependiendo del tipo de situaciones; por ejemplo, cuando se piensa en el futuro, las decisiones de líneas estratégicas para la inversión en recursos de nuevos productos o servicios son siempre complicadas, y más aún cuando se necesita justificar las inversiones en el mercado.

En segundo lugar, las decisiones de gestión normalmente están asociadas al tipo y calidad de recursos, otra situación se presenta cuando se considera el impacto de una decisión más táctica, mientras más importantes son los recursos, más complejo es el impacto de la decisión; pero también hay decisiones con pequeñas inversiones de recursos que generan gran impacto en los resultados, en dos sentidos: ya sea para buenos resultados o para malos resultados; sin embargo, no todo está en función a la inversión, además se pueden evaluar criterios de calidad y de oportunidad, entre otros.

En tercer lugar, las decisiones operativas más comunes van desde la elaboración de una memoria empresarial que guíe el camino de mediano o largo plazo, pasando por las listas de información especializada para fines específicos, por ejemplo cuando se requiere conocer la trazabilidad sobre nuestras decisiones en cambios de precios o servicios, o también por obtener el conocimiento de las necesidades del mercado en sus diferentes enfoques, hasta completar la necesidad de entregar información de las experiencias y vivencias de las personas experimentadas a los nuevos miembros de las organizaciones o aquellos que deben desempeñar su trabajo en zonas geográficas aisladas, o en la toma de decisiones complejas.

Es común escuchar hablar de los sistemas que procesan información como sistemas de gestión documental, donde el objetivo es grabar la información y tener los mecanismos adecuados para recuperar la información previamente 
almacenada. Pero hoy en día eso ya no es suficiente, debido a la gran cantidad de datos que se disponen en los sistemas, y a la gran cantidad de información que está disponible en Internet, y la gran cantidad de posibles interpretaciones; por lo cual es relevante el correcto entendimiento de los problemas difusos de interpretación ambigua; donde la ambigüedad fue ocasionada por la globalización, la diversidad geográfica o los productos y servicios adquiridos de países con otras costumbres; por ejemplo, la sensación de frío es una percepción diferente para una persona que vive en la costa que para una persona que vive en la sierra. Lo mismo ocurre en los contextos empresariales; por ejemplo, en la minería el agua de una fuente o pequeño lago debe ser retirada para extraer los minerales preciosos, pero la misma fuente de agua constituye una propiedad de alto valor para los pobladores, así no puedan consumir nunca el agua, y de la misma forma se pueden encontrar varios ejemplos de un mismo elemento que tienen distintas perspectivas de valor para un empresario, un trabajador o un ciudadano, y estas diferencias tienen impacto directo en la rentabilidad de la empresa, ya sea que se trate de una rentabilidad económica o social, debido a las divergencias generadas entre las partes. En resumen, la información atraviesa un nuevo escenario de gran cantidad de datos disponibles nunca antes visto, y de la oportunidad de entender las diferentes perspectivas de las personas en torno a un mismo problema. Lo anterior permite comprender lo señalado por Saiz y Olalla:

La obtención de una información transparente por parte de los stakeholders de una organización es fundamental para que la misma logre unas elevadas tasas de crecimiento sostenible en el tiempo. La razón de este hecho viene dado por la importancia que tienen, tanto clientes como stakeholders (principalmente accionistas), en la generación de flujos de caja (cash flows) presentes y futuros, para lograr así la supervivencia de la organización (2010, p. 73).

Se puede afirmar, tal como lo indican los autores citados, que "el valor asociado al recurso de la información y el conocimiento es incalculable en una sociedad de la información" (Saiz y Olalla, 2010); y además, es necesario tomar conciencia de que las empresas deben disponer de un sistema de gestión del conocimiento y de la información que se adecue a sus necesidades y características empresariales y de la información que procesa.

Por ejemplo, ¿cuántas veces se debe volver a ejecutar un proceso, un informe, o simplemente corregir un error en el presupuesto? Un error en el presupuesto lleva a invertir dinero para adquirir o alquilar un lugar de trabajo adecuado, pero luego se necesita más dinero para hacerlo funcionar, y normalmente pasa que no se tiene la información correcta de los costos involucrados, porque eso significa un trabajo adicional y un esfuerzo y dedicación en tiempo que muchas veces no se realiza. Entonces, se tiene un problema: se toman decisiones sin la información mínima o adecuada para medir el impacto futuro de las decisiones adoptadas; lo 
que es perjudicial para una actividad productiva en cualquier campo o sector de la industria o del Estado.

Figura 1. Representación de grandes volúmenes de información

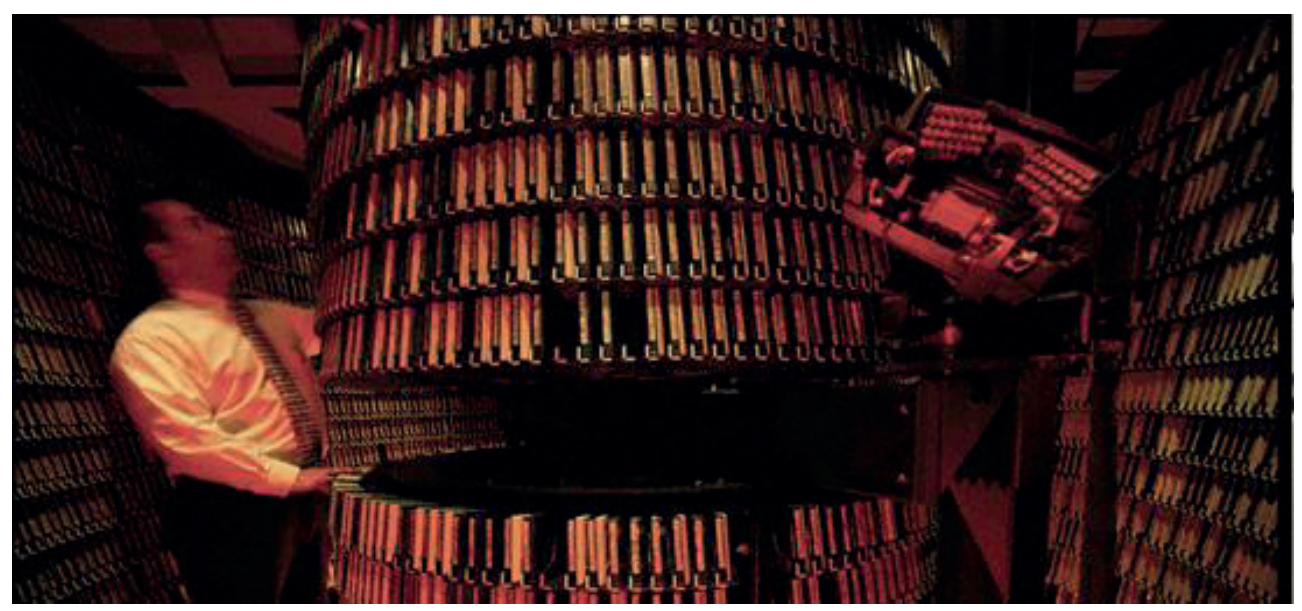

Fuente: Boston University Metropolitan College.

A continuación se presenta un resumen de las características de la información que es útil para las empresas (Saiz y Olalla, 2010):

a) Tendencia a ampliar los nichos de mercado y posibilidades de nuevos negocios.

b) Formación especializada dentro de la organización.

c) Disposición hacia el pensamiento y experiencia poco convencional.

d) Grado de confianza entre las personas.

\section{Definiciones de la gestión del conocimiento}

Tal como señala Alavi y Leidner (2001), en relación a la gestión del conocimiento:

Aunque el concepto de codificar, almacenar y transmitir conocimiento en las organizaciones no es nuevo, dado que se conocen programas de entrenamiento, de desarrollo, de políticas, procedimientos (Alavi y Leidner, 1999), la práctica de gestión organizacional tiene un enfoque reciente en el conocimiento, por ejemplo el benchmarking, buenas prácticas, conocimiento organizacional y otros activos intangibles en general (Grant, 1996) por ello se debe entender la gestión del conocimiento y los sistemas de gestión del conocimiento en las organizaciones (p. 108). 
De lo señalado se aprecia que es necesario que las organizaciones entiendan estos conceptos. A continuación se presentan algunas definiciones de gestión del conocimiento:

Tabla 1. Definiciones de gestión del conocimiento

\begin{tabular}{|c|c|c|}
\hline & Autor & Definición \\
\hline 1 & $\begin{array}{l}\text { Davenport \& } \\
\text { Prusak } \\
\text { (1998) }\end{array}$ & $\begin{array}{l}\text { Se modela a partir de los recursos existentes que su organización } \\
\text { puede tener en buenos sistemas de información gerencial, gestión } \\
\text { del cambio organizacional y gestión de los recursos humanos. }\end{array}$ \\
\hline 2 & $\begin{array}{l}\text { Swan et al. } \\
(1999)\end{array}$ & $\begin{array}{l}\text { Cualquier proceso o práctica de crear, adquirir, capturar, compartir y } \\
\text { utilizar el conocimiento, donde reside, para mejorar el aprendizaje y } \\
\text { desempeño en las organizaciones. }\end{array}$ \\
\hline 3 & Skyrme (1999) & $\begin{array}{l}\text { La gestión sistemática de conocimiento vital asociado a procesos de } \\
\text { creación, recopilación, organización, difusión, uso y explotación, en } \\
\text { la búsqueda de objetivos de la organización. }\end{array}$ \\
\hline 4 & $\begin{array}{l}\text { Mertins et al. } \\
(2000)\end{array}$ & $\begin{array}{l}\text { Los métodos, instrumentos y herramientas que en un enfoque ho- } \\
\text { lístico contribuyen a la promoción de los conocimientos base (core } \\
\text { knowledge) de los procesos. }\end{array}$ \\
\hline 5 & $\begin{array}{l}\text { uit Beijerse } \\
(2000)\end{array}$ & $\begin{array}{l}\text { El logro de las metas de la organización hechas por el factor de co- } \\
\text { nocimiento productivo. }\end{array}$ \\
\hline 6 & $\begin{array}{l}\text { Newell et al. } \\
(2002)\end{array}$ & $\begin{array}{l}\text { La mejora de las formas como las empresas enfrentan entornos al- } \\
\text { tamente turbulentos puede movilizar a su base de conocimientos } \\
\text { (o aprovechar su conocimiento «Activos») con el fin de asegurar la } \\
\text { innovación continua. }\end{array}$ \\
\hline
\end{tabular}

Fuente: University of London, 2014, p. 7.

Es necesario tener una comprensión de los problemas que deben enfrentar las empresas y organizaciones para gestionar el conocimiento en forma de información y experiencias. Tal como señala Jashapara, desde una perspectiva interdisciplinaria la gestión del conocimiento puede definirse como: "Los procesos efectivos de aprendizaje asociados con la exploración, explotación e intercambio del conocimiento humano (tácito y explícito) que utiliza la tecnología apropiada y los entornos culturales para mejorar el rendimiento y el capital intelectual de las organizaciones" (citado por University of London, 2012, p. 8).

Existe una variedad de definiciones de la gestión del conocimiento, en la medida en que las organizaciones y sus perspectivas son diferentes, por lo que se concluye que la gestión del conocimiento se puede entender de muchas maneras para diferentes disciplinas o sectores de la actividad productiva. Por ejemplo, para 
las ciencias de la comunicación, ciencias sociales y humanidades, el conocimiento es la información que existe en un documento escrito, o en la experiencia de unas personas; entonces, la gestión del conocimiento es compartir la información o las experiencias con otras personas.

En el ámbito de la seguridad ciudadana, la gestión del conocimiento gira en torno a mantener la información aprendida disponible para otras personas; suele suceder que se realizan determinadas actividades sin tomar las precauciones necesarias; por ejemplo, se deja la puerta abierta de la casa o del auto por algún breve momento y cuando menos se espera sucede un robo o asalto. En otras ocasiones se sale de viaje por unos días y se deja la casa abandonada, y cuando se regresa algo malo ha sucedido. Entonces surgen las lamentaciones, pero se aprende la lección, y no se repite el hecho, y más aún: se adoptan algunas medidas adicionales de seguridad, como colocar candados, alarmas, etcétera. Luego se informa de lo ocurrido a las amistades y a los familiares, y se publica en las redes sociales con la finalidad de que no le suceda lo mismo a otras personas. La misma idea de compartir información y experiencias sucede cuando se ha tenido una buena experiencia en un restaurante o en un viaje, se narra lo sucedido e incluso se brindan recomendaciones para que los demás también lo disfruten. Este círculo es la gestión del conocimiento.

También existe la gestión del conocimiento en la medicina y en otras actividades que requieren de la experiencia previa. La situación es similar pero más específica, se requiere compartir la experiencia previa pero ahora con nuevas personas, nuevos pacientes, nuevos diagnósticos, y tal vez todos unidos en una nueva combinación de eventos. Por ejemplo, si un médico experimentado no encuentra la causa de una fiebre, emite diagnósticos y predicciones, como el “Dr. House", hasta que encuentra la causa y se identifican las variables que sobre la base del diagnóstico se produce un tratamiento y un resultado, el cual se espera sea positivo. Luego, una situación similar puede repetirse en el futuro con otras personas, pero se debe confirmar que se cumplan todas las condiciones similares al problema anterior; por ejemplo, si el mal se contrajo en algún lugar tropical, el mismo evento debe estar presente en el nuevo caso, si no se debería dudar razonablemente del diagnóstico. Pero, ¿cómo se hace si el primer caso sucedió en un país de Centroamérica y el segundo ha sucedido en América del Sur; o tal vez el primer caso sucedió en la selva peruana y el segundo caso está sucediendo en la ciudad de Lima? Si se logra hacer que la información y la experiencia lleguen a su destino, el lugar donde se necesita y a tiempo, se puede decir que se ha logrado la gestión del conocimiento. 
En ingeniería suceden muchos casos de experiencias y toma de decisiones asociadas a diseños de obras civiles, en la actividad minera, en la construcción y en la operación de naves para el aire o el agua, donde el costo de repetir un error es muy alto, y es mejor aprender de las experiencias y correcciones realizadas con éxito ante un desafío de la empresa.

En las investigaciones llevadas a cabo en la universidad el desafío radica en analizar y entender situaciones difíciles y complejas de nuestra sociedad local o global, en contextos multidisciplinarios; entender los esfuerzos, los métodos y las técnicas que se han venido realizando para solucionar problemas y luego proponer nuevas formas de solucionarlos; por ello muchas empresas o instituciones de gobierno apoyan las investigaciones serias en las universidades de los países desarrollados.

Como se puede apreciar, es fundamental entender que se deben adoptar diferentes perspectivas interdisciplinarias en las distintas actividades productivas y de valor en la sociedad, para enfocar la gestión del conocimiento. El siguiente paso es entender las clasificaciones que se presentan en el conocimiento y las TIC.

Como señalan Alavi y Leidner, "el conocimiento es definido para justificar la creencia que es posible incrementar la capacidad empresarial y la capacidad organizacional por medio de acciones efectivas (2001)". Las clasificaciones mostradas por los autores citados incluyen:

a) conocimiento vía datos e información

b) el estado de la mente

c) un objeto

d) un proceso

e) una condición para tener acceso a información

f) capacidades.

Estas posibilidades tienen diferentes requisitos y van a ocasionar distintos impactos en las empresas y las organizaciones. En la tabla 2 se presenta la clasificación elaborada por Alavi y Leidner. 
Tabla 2. Perspectivas del conocimiento y sus implicaciones

\begin{tabular}{|c|c|c|c|c|}
\hline & Perspectiva & Descripción & $\begin{array}{c}\text { Implicaciones } \\
\text { para la gestión del } \\
\text { conocimiento }\end{array}$ & $\begin{array}{c}\text { Implicaciones para los } \\
\text { sistemas de gestión del } \\
\text { conocimiento }\end{array}$ \\
\hline 1 & $\begin{array}{l}\text { Datos, infor- } \\
\text { mación y co- } \\
\text { nocimiento }\end{array}$ & $\begin{array}{l}\text { Dato es hecho, in- } \\
\text { formación es dato } \\
\text { procesado o inter- } \\
\text { pretado. } \\
\text { Conocimiento es } \\
\text { información perso- } \\
\text { nalizada }\end{array}$ & $\begin{array}{l}\text { Uso potencial de in- } \\
\text { formación, facilita la } \\
\text { asimilación de infor- } \\
\text { mación }\end{array}$ & $\begin{array}{l}\text { Es parecido a un SIG } \\
\text { pero extendido para } \\
\text { ayudar el uso y asimila- } \\
\text { ción de la información }\end{array}$ \\
\hline 2 & $\begin{array}{l}\text { Estado de la } \\
\text { mente }\end{array}$ & $\begin{array}{l}\text { Conocimiento es el } \\
\text { estado del conocer } \\
\text { y entender }\end{array}$ & $\begin{array}{l}\text { Envuelve el aprendiza- } \\
\text { je individual con la pro- } \\
\text { visión de información }\end{array}$ & $\begin{array}{l}\text { Provee acceso a fuen- } \\
\text { tes de conocimiento } \\
\text { más que el conoci- } \\
\text { miento mismo }\end{array}$ \\
\hline 3 & Un objeto & $\begin{array}{l}\text { Conocimiento es un } \\
\text { objeto que puede } \\
\text { ser almacenado y } \\
\text { manipulado }\end{array}$ & $\begin{array}{l}\text { Construye activos para } \\
\text { gestionar el conoci- } \\
\text { miento }\end{array}$ & $\begin{array}{l}\text { Desarrolla la obtención, } \\
\text { almacenamiento y } \\
\text { transferencia del cono- } \\
\text { cimiento }\end{array}$ \\
\hline 4 & Un proceso & $\begin{array}{l}\text { Conocimiento es un } \\
\text { proceso de aplica- } \\
\text { ción de experiencias }\end{array}$ & $\begin{array}{l}\text { Enfoca el flujo del co- } \\
\text { nocimiento y los pro- } \\
\text { cesos de crear, com- } \\
\text { partir y distribuir cono- } \\
\text { cimiento }\end{array}$ & $\begin{array}{l}\text { Provee unión entre } \\
\text { las fuentes de conoci- } \\
\text { miento para crear en- } \\
\text { tendimiento y flujo del } \\
\text { conocimiento }\end{array}$ \\
\hline 5 & $\begin{array}{l}\text { Acceso a in- } \\
\text { formación }\end{array}$ & $\begin{array}{l}\text { Conocimiento es } \\
\text { una condición de } \\
\text { acceso a informa- } \\
\text { ción }\end{array}$ & $\begin{array}{l}\text { Enfoca el acceso orga- } \\
\text { nizado y recuperación } \\
\text { de contenidos }\end{array}$ & $\begin{array}{l}\text { Provee búsqueda efec- } \\
\text { tiva y mecanismos para } \\
\text { localizar información } \\
\text { relevante }\end{array}$ \\
\hline 6 & Capacidades & $\begin{array}{l}\text { Conocimiento es el } \\
\text { potencial de accio- } \\
\text { nes influyentes en } \\
\text { el resultado }\end{array}$ & $\begin{array}{l}\text { Construye competen- } \\
\text { cias core para entender } \\
\text { el estratégico know- } \\
\text { how }\end{array}$ & $\begin{array}{l}\text { Mejora el capital inte- } \\
\text { lectual, desarrollando } \\
\text { competencias indivi- } \\
\text { duales y organizacio- } \\
\text { nales }\end{array}$ \\
\hline
\end{tabular}

Fuente: Alavi y Leidner, 2001.

También existen muchas situaciones que parecen simples pero que son muy importantes, como el flujo de un procedimiento correcto y el hecho de tener un informe importante a la mano. 
Figura 2. Modelo para compartir información

\section{Compartir información y experiencias personales}

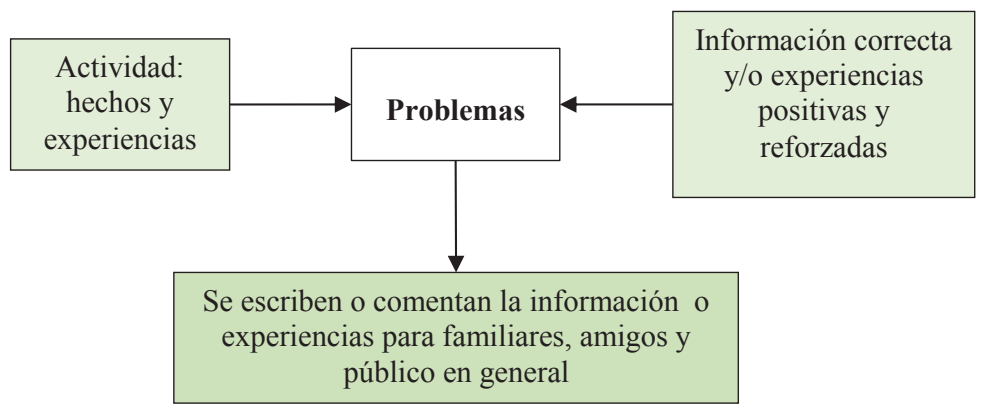

Elaboración propia.

En un primer caso parece evidente que en la empresa todos deben saber cuáles son los procedimientos establecidos para las diferentes situaciones, por ejemplo para contratar personas o para aprobar una compra; sin embargo, hay casos como el de un nuevo trabajador que ingresa a laborar en la empresa y debe comenzar inmediatamente, siendo normal que no conozca todos los procedimientos y que algunos pasos no se cumplan; o cuando en una época especial, y por ampliación de locales, se realizó un estudio de costos y de riesgos, y la persona que lo hizo tenía 20 años de experiencia pero se fue de la empresa, y se tiene que hacer un trabajo similar pero el nuevo trabajador no sabe por dónde empezar, y tal vez ni siquiera sepa que se requiere el estudio.

La experiencia de una persona con 20 o 30 años de trabajo en la compañía no es un problema mientras esa persona trabaja en la empresa, pero cuando se requiere resolver un problema similar en otra ciudad, donde viajar genera un costo muy alto en tiempo, dinero y recursos por tratarse de una zona geográficamente aislada, o cuando la persona experimentada se retira o deja la empresa por cualquier motivo, se vuelve una urgencia y una emergencia contar con su conocimiento. Entonces, una empresa tiene muchas necesidades de guardar la información importante de sus actividades, ya sea para decisiones de gestión o para decisiones operativas. Las decisiones de gestión son variadas cuando se piensa en el futuro; por ejemplo, en decisiones de líneas estratégicas para la inversión en recursos de nuevos productos o servicios cuando se necesita justificar las inversiones en el mercado.

Las decisiones operativas son variadas, desde la elaboración de una memoria empresarial que guíe el camino de mediano o largo plazo, pasando por las listas de información especializada para fines específicos; por ejemplo, cuando se requiere conocer el impacto o los reclamos sobre nuestras decisiones en cambio 
de precio o servicios, pasando por la necesidad de tener el conocimiento de los requerimientos del mercado en sus diferentes enfoques, hasta completar la entrega de información de las personas experimentadas a los nuevos o a aquellos que se encuentran en zonas geográficas aisladas.

Ahora suponga que todas estas formas de desarrollar la gestión del conocimiento deben transmitirse, ya sea en forma oral, escrita o por medios digitales, y surge la pregunta sobre cuál es el rol de la tecnología para mejorar estas formas de gestionar información; si se piensa en el rol de la ingeniería de sistemas, ya sea para la información generada en el ámbito empresarial o social, se sabe que es necesario aplicar un enfoque holístico para la gestión de la información.

En ingeniería de sistemas y las áreas relacionadas con las tecnologías de información se deben utilizar las tecnologías de hardware, software y comunicaciones para gestionar de forma eficiente los procesos y generar nuevas modalidades para el desarrollo de los proyectos. Es aquí donde la gestión del conocimiento adquiere una nueva dimensión —una dimensión tecnológicay empieza la nueva historia, desde los antiguos procesos de sistemas expertos de los años setenta hasta las nuevas tecnologías de colaboración basadas en agentes inteligentes y en Internet. Se puede realizar una primera conclusión sobre los tipos de gestión del conocimiento en un enfoque de ingeniería de sistemas, donde existe un rol empresarial y una cadena de valor, o donde existe un sector productivo y varios sistemas y subsistemas interrelacionados, entonces, se puede hacer una propuesta para los nuevos sistemas de gestión del conocimiento como:

1) Compartir información

2) Compartir experiencias

3) Análisis de problemas simples

4) Análisis de problemas complejos

5) Solución a objetivos empresariales

6) Diseño de toma de decisiones mediante sistemas inteligentes, llamados agentes.

\section{7) Diseño de sistemas multiagentes adaptativos}

Como se aprecia, las clasificaciones de compartir información y de compartir experiencias tienen muchas utilidades reales y muchas oportunidades de uso en diferentes disciplinas o sectores productivos, donde en realidad no se requieren sistemas sofisticados sino que basta con una base de datos y unos sistemas tipo web o blog en Internet. Aquí lo importante es tener estos 
sistemas actualizados, porque la información desactualizada no tiene el valor que se espera recibir.

Un nivel de mayor importancia lleva a analizar los diferentes problemas en una empresa y sus soluciones, donde se desarrollan el análisis de problemas simples, la solución a objetivos empresariales y el análisis de problemas complejos.

Primero se analizarán algunos de los niveles de complejidad conocidos en una empresa; los procesos se pueden clasificar en:

i. Procesos de nivel bajo de complejidad. Son los procesos administrativos, por ejemplo en los trámites de la rutina diaria, donde existe un procedimiento que debe ser conocido y las excepciones son resueltas con el sentido común.

ii. Procesos de nivel medio de complejidad. Requieren de un nivel de decisión y aprobación, por ejemplo en las decisiones sobre la contratación o despido de personal, en los pedidos y/o solicitudes que involucran una inversión, decisiones de gasto en actividades no previstas, oportunidad financiera o riesgo financiero. Procesos de aprendizaje y perfiles de usuarios.

iii. Procesos de nivel alto de complejidad. Requieren de más de un nivel de decisión, o la decisión es de gran impacto para la vida o para las finanzas, por ejemplo en la solución de un problema médico, la inversión en la bolsa de valores, en la selección de cursos que se dictarán en un semestre, evaluación de resultados académicos y financieros, gestión en inversión de proyectos, alianzas y sociedades estratégicas. Estas actividades son complejas, y si se analizan en forma conjunta con los tipos de gestión del conocimiento se podrá construir una matriz de complejidad.

Cuando se busca un ejemplo de la gestión del conocimiento relacionado a una empresa, o una organización se puede establecer que ya existen sistemas, proyectos e investigaciones desarrolladas en alguna parte del mundo, y que estos se presentan en los mejores congresos de Knowledge Management en el ámbito global. Es así que para analizar un sistema de gestión del conocimiento se puede pensar en diferentes formas, ya sea para compartir información, compartir experiencias o solucionar problemas complejos; en este último caso es recomendable simular un sistema, paso a paso, incluyendo a todos los actores, desde que ocurre la necesidad hasta que el problema es atendido por el sistema, en otras palabras, ir modelando las transacciones que se ejecutarán en el sistema. 


\section{La gestión del conocimiento y las TIC}

Nuestro entorno es la empresa y las TIC, nuestra visión en el mundo es trabajar en forma colaborativa con las personas, organizaciones y países a través de las TIC para promover un bienestar común junto con la integración de la persona en la sociedad de la información y el conocimiento. Se quiere superar los grandes problemas que afrontaron los proyectos de software, desde su aparición en la década de 1950 hasta nuestros días, y sumarse a los esfuerzos que a partir de problemas diversos se hacen en función de la gestión exitosa de proyectos, entre ellos la gestión de sistemas colaborativos.

Existe el deseo de adaptarse y cambiar de forma continua con el entorno, además de ser parte de los actores que promueven los cambios para obtener ventajas competitivas y posicionamiento tecnológico a través de las Tl y el aprendizaje continuo. Se busca ser creativos, en innovaciones constantes, en una visión compartida, en una organización que aprende. Para lograrlo se deben integrar los procesos estratégicos, tácticos y operativos para una correcta y efectiva dirección. En estas nuevas estrategias el entorno juega un papel muy importante, tal como señala Paniagua:

es un entorno complejo, ya que es un entorno global; hay diversificación de los mercados, ya que la organización, para aumentar su competitividad, debe abrir el abanico de sus productos y ofertarlos a diferentes mercados objetivo (ubicados en cualquier lugar del mundo); es dinámico, al ser un mercado mucho más amplio, existe diversidad de comportamientos y reacciones no predecibles con facilidad; y de vez en cuando, presenta hostilidad a la organización, provocando transformaciones en sus mecanismos de coordinación y estructura (2007, p. 20).

A ello se deben añadir los problemas sociales, de inmigración y de envejecimiento en el mundo; la falta de colaboración y el cruel día a día de las organizaciones que muchas veces limita los planes a largo plazo.

El conocimiento es información, reglas y experiencia que se deben usar en un contexto familiar, social, profesional, educativo, en una organización, cuando se toman decisiones. El conocimiento existe en las personas, y son trasladadas a experiencias, documentos, aplicaciones, bases de datos, sistemas inteligentes, etcétera. Según Nonaka y Takeuchi (1997), la gestión del conocimiento puede definirse como "la capacidad orgánica para generar nuevos conocimientos, diseminados entre los miembros de una organización y materializarlos en productos, servicios y sistemas. Es la clave del proceso a través del cual las firmas innovan" (citados en Bernuy, 2005). También se define como el desarrollo de la 
gestión estratégica en las siguientes áreas, definidas por Blanchart (2000), tal como se cita en Bernuy (2005):
a) gestión de la información
b) gestión de inteligencia
c) gestión de documentación
d) gestión de recursos humanos
e) gestión de innovación y cambio
f) organización del trabajo.

Entonces, se entiende que gestionar conocimiento es plantear y aplicar nuestras capacidades para lograr el éxito en cuatro fases:

(1) Obtener el conocimiento esencial en nuestra organización;

(2) Almacenarlo en forma correcta y organizada; para

(3) Poder diseminarlo; y

(4) Finalmente, usarlo eficientemente.

Los sistemas de gestión del conocimiento, tal como señalan Salinas, De Benito, Marín, Moreno y Morales: "Canals (2008) considera que 'un sistema de gestión del conocimiento comprende todo aquello que contribuye a facilitar los procesos de creación y transmisión de conocimiento, así como su utilización'"' (2010, p. 2).

Asimismo, Alavi y Leidner (2001), tal como citan Salinas et al. (2010), describen los sistemas de gestión del conocimiento como sistemas aplicados a gestionar el conocimiento organizacional, donde se busca mejorar los procesos de creación, almacenamiento, recuperación, transferencia y uso del conocimiento.

El punto de partida de ambos autores implica la creación del conocimiento organizacional para su posterior gestión. Actualmente existen herramientas y técnicas que han sido utilizadas en parte o en forma completa como sistemas de gestión del conocimiento, pero dado que se trata de un campo en evolución se deben tener los criterios adecuados para aceptar el progreso de los sistemas en el tiempo. Una clasificación de herramientas y técnicas se muestra en la tabla 3: 
Tabla 3. Algunas herramientas y técnicas de gestión del conocimiento

\begin{tabular}{|c|c|}
\hline Autor & Ejemplos \\
\hline $\begin{array}{l}\text { Núñez } \\
\text { Paula, l.; } \\
\text { Núñnez } \\
\text { Govín, Y. } \\
\text { (2006) }\end{array}$ & $\begin{array}{l}\text { a) Herramientas de búsqueda y recuperación de la información: motores de búsqueda } \\
\text { y metabuscadores. } \\
\text { b) Herramientas de filtrado y personalización de la información. } \\
\text { c) Tecnologías de almacenamiento y organización de la información: sistemas de } \\
\text { gestión de bases de datos, data warehousing, asignación de metadatos. } \\
\text { d) Herramientas de análisis de información: minería de datos, minería de textos, ár- } \\
\text { boles de decisión y sistemas expertos, razonamiento basado en casos, tecnologías } \\
\text { de autoorganización. } \\
\text { e) Sistemas de gestión de flujos y comunicación: representación de diagramas de } \\
\text { flujos de datos o herramientas CASE, elaboración de mapas conceptuales o de } \\
\text { conocimiento, comunicación y elaboración grupal, flujo de trabajo. } \\
\text { f) Herramientas de aprendizaje y comercio electrónico (sistemas de e-learning y } \\
\text { e-commerce). } \\
\text { g) Sistemas de gestión empresarial. }\end{array}$ \\
\hline $\begin{array}{l}\text { Maier, R. } \\
\text { (2007) }\end{array}$ & $\begin{array}{l}\text { a) Organizacional: sistemas de broadcasting, repositorios de conocimiento, portales de } \\
\text { conocimiento corporativo, servicios de directorio, sistemas de metabúsqueda, sis- } \\
\text { temas de conocimiento push (suscripciones de información, soporte de la comuni- } \\
\text { dad), sistemas de visualización de conocimiento (mapas de conocimiento), soporte } \\
\text { de la comunidad del proceso de trabajo, paquetes e-learning, agentes inteligentes } \\
\text { para el soporte del procesamiento de la información organizacional. } \\
\text { b) Grupo y comunidad: construcción de comunidades y espacios de trabajo, sistemas } \\
\text { de gestión del flujo de trabajo, sistemas de comunicación múltiple (listas de ser- } \\
\text { vidores, grupos de noticias, videoconferencia de grupo), sistemas de colaboración, } \\
\text { agentes inteligentes apoyando el procesamiento de la información en grupos. } \\
\text { c) Individual: sistemas de búsqueda personal (perfiles de usuarios, filtros de búsqueda), } \\
\text { descubrimiento de conocimiento y mapeado, sistemas de comunicación de uno a } \\
\text { uno (email, videoconferencia de uno a uno, mensajería instantánea), agentes inteli- } \\
\text { gentes para la gestión del conocimiento personal. }\end{array}$ \\
\hline $\begin{array}{l}\text { Grau, A. } \\
(2002)\end{array}$ & $\begin{array}{l}\text { a) Herramientas de búsqueda y clasificación de la información. } \\
\text { b) Colaboración, trabajo en grupo, conferencias, e-mail y mensajería. } \\
\text { c) Herramientas de simulación. } \\
\text { d) Portal corporativo. } \\
\text { e) Filtrado y distribución personalizada de información. }\end{array}$ \\
\hline $\begin{array}{l}\text { Beatriz } \\
\text { Peluffo A., } \\
\text { M.; Catalán } \\
\text { Contreras, } \\
\text { E. (2002) }\end{array}$ & $\begin{array}{l}\text { a) Administración de conocimientos: gestión de contenido, administración de docu- } \\
\text { mentos, bases de datos, manejo de información y búsqueda. } \\
\text { b) Apoyo a la creación y el aprendizaje: motores de búsqueda, acceso a conocimiento } \\
\text { experto, generadores de ideas, mapas mentales, espacios de aprendizaje, espacios } \\
\text { de conversación. } \\
\text { c) Visualización: Internet, intranet, extranet y portales }\end{array}$ \\
\hline
\end{tabular}

Fuente: Adaptado de Salinas et al., 2010, p. 3. 
Se puede apreciar que todos los esfuerzos realizados han sido dirigidos a la administración de contenidos, pero ninguno en el sentido de gestionar los procesos de creación del conocimiento organizacional.

\section{Sistemas de gestión del conocimiento}

Pavez (2000) define la organización inteligente como:

la capacidad de la empresa, con independencia de su tamaño, características y posición competitiva en su sector, para procesar conocimiento para conseguir soluciones óptimas que permitan a la misma lograr su supervivencia en entornos competitivos. Se puede hablar así del concepto de organización inteligente en el mercado actual, donde su mayor potencial radica en la capacidad de aprender (citado en Saiz y Olalla, 2010, p. 74).

De lo cual se comprende —y desde la perspectiva de la ingeniería de sistemasque el conocimiento es el aprendizaje dinámico que una persona, dentro de una organización, puede entender como el resultado de la toma de decisiones en un proceso productivo determinado o de un hecho vivido dentro de dicho proceso. Este aprendizaje dependerá de la perspectiva del mundo de cada persona.

De acuerdo con lo que señalan Bratinau, Vasilache y Jianu (2006), "La característica distintiva de las organizaciones inteligentes viene dada por su capacidad de adaptación en entornos cambiantes gracias a una nueva estructura cognitiva formada por el capital intelectual de la organización" (citado en Saiz y Olalla, 2010, p. 74).

Con lo que se puede afirmar que la capacidad de adaptación está en las personas, y su capital intelectual parte de ellas, quienes buscan crear nuevos procesos, optimizar los procesos actuales y mejorar las tomas de decisiones en los diferentes problemas que tengan que afrontar, evitando los riesgos de impactos negativos como resultado de un proceso determinado.

Estos procesos han ocurrido en un contexto, con unos actores, unas variables basadas en una experiencia previa, y con información necesaria y pertinente para el aprendizaje; ya sea por la toma de decisiones o por la asimilación del hecho vivido se adquiere conocimiento. De esta forma, se requiere de un criterio y un enfoque adecuado y mientras más información se disponga mejor será la toma de decisiones y mejor será el resultado esperado. Si se puede explicar el proceso de creación del conocimiento asociado al proceso productivo, entonces el conocimiento será explícito, de otra forma será conocimiento tácito. 
De acuerdo con Saiz y Olalla:

Una de las ventajas competitivas más relevantes para la empresa es la de preparar el escenario adecuado para la correcta, ágil y fundamentada toma de decisiones a nivel estratégico. Esto aportará la ventaja competitiva en la flexibilidad como en la adaptación a los cambios y necesidades del mercado en cada momento (2010, pp. 74-75).

Por todo ello, el objetivo de los sistemas de gestión del conocimiento es apoyar la creación, transferencia y aplicación del conocimiento en las organizaciones, en diferentes momentos pero con mayor impacto en la toma de decisiones de problemas complejos. Cuando el conocimiento es parte de un proceso se debe gestionar el flujo de información, así como las formas de crear, compartir y distribuir el conocimiento hasta su uso.

Figura 3. Modelo conceptual de la creación del conocimiento

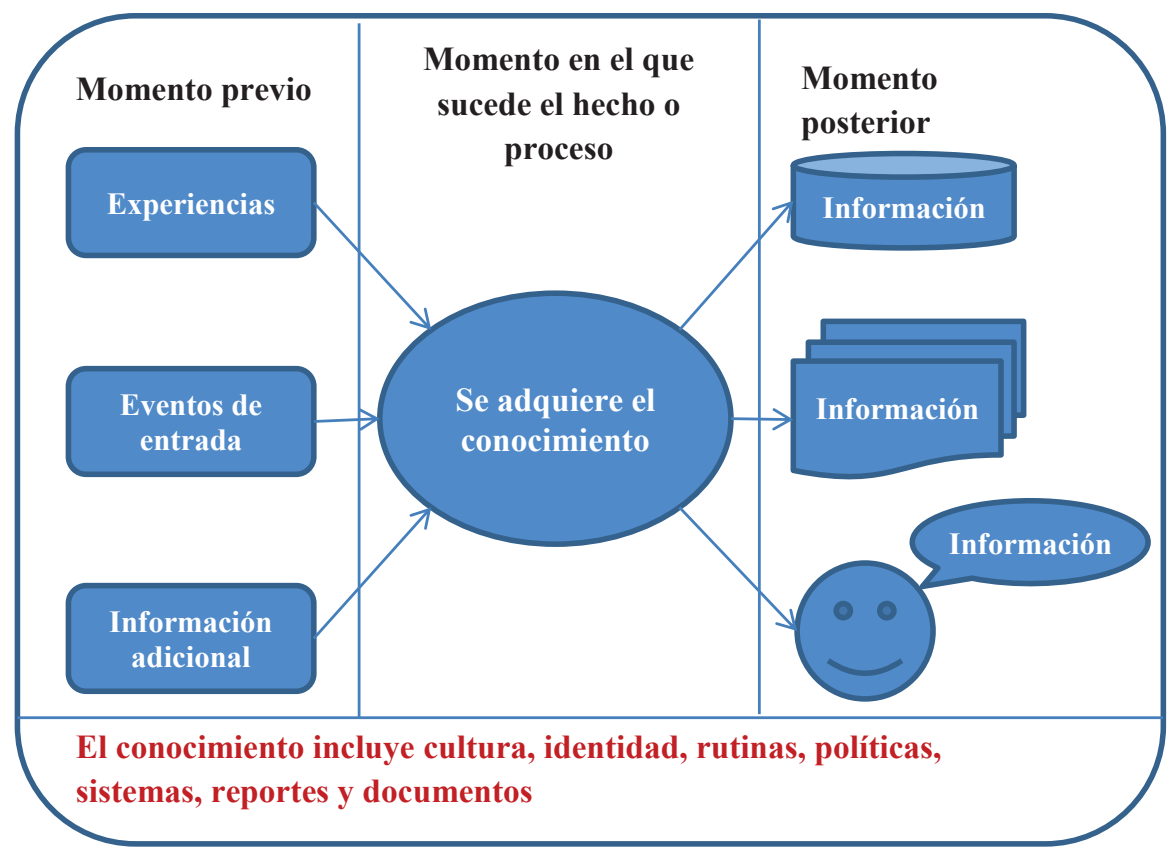

Elaboración propia.

Es así como se definen los modelos que permitirán afrontar cambios estratégicos en las empresas y las organizaciones, implementando sistemas de gestión del conocimiento. Los ejemplos incluyen encontrar la base de datos de un experto o 
una fuente de conocimiento existente, utilizando directorios en línea y buscando bases de datos; además de compartir el conocimiento y trabajando en equipos virtuales, para tener acceso a información de situaciones anteriores, al aprendizaje de las necesidades y el comportamiento de los usuarios o clientes.

El modelo capaz de enfrentar este desafío debe entender primero que el momento en que el conocimiento aparece es en un tiempo muy corto, donde el cerebro humano razona a gran velocidad, aún no conocida por la ciencia, y es en esa fracción de tiempo que se deben identificar los macroprocesos que deben suceder.

\section{Sistemas de gestión del conocimiento colaborativos}

Los sistemas de gestión del conocimiento colaborativos se basan en el soporte a los procesos de creación, almacenamiento, recuperación, transferencia y aplicación del conocimiento, unido a las tecnologías e-business. En relación con las nuevas tecnologías, Siemens (2014) anota:

gracias al desarrollo de una nueva generación de soluciones que unifican sistemas operativos, automatizan plataformas productivas y permiten optimizar, así, los procesos de fabricación. Las soluciones de software e IT, los sistemas de control y los productos innovadores de Siemens incrementan la productividad de la industria y su competitividad, a la vez que reducen significativamente los costes y el uso eficiente de los recursos y flexibilizan los recursos.

El objetivo de los sistemas de gestión del conocimiento es apoyar la creación, transferencia y aplicación del conocimiento en las organizaciones. Cuando el conocimiento es parte de un proceso se debe gestionar el flujo de información, así como las formas de crear, compartir y distribuir el conocimiento. Luego se debe preparar un modelo tecnológico que afronte este contexto.

Aquí se logra el desafío de gestionar la creación del conocimiento organizacional, se modela una solución con componentes de tecnología de avanzada como análisis de datos, agentes de software, sistemas GPS, tecnología M2M y, además, debe estar alineada a la arquitectura empresarial, con interfaces de usuario, de forma que la solución pueda ser accionada oportunamente y se logre el objetivo de resolver la toma de decisiones de problemas complejos. Es necesario señalar que si bien está incluido dentro de un enfoque e-business, existen otras componentes conceptuales para su integración con el capital intelectual. 
Figura 4. Modelo sistema de gestión del conocimiento

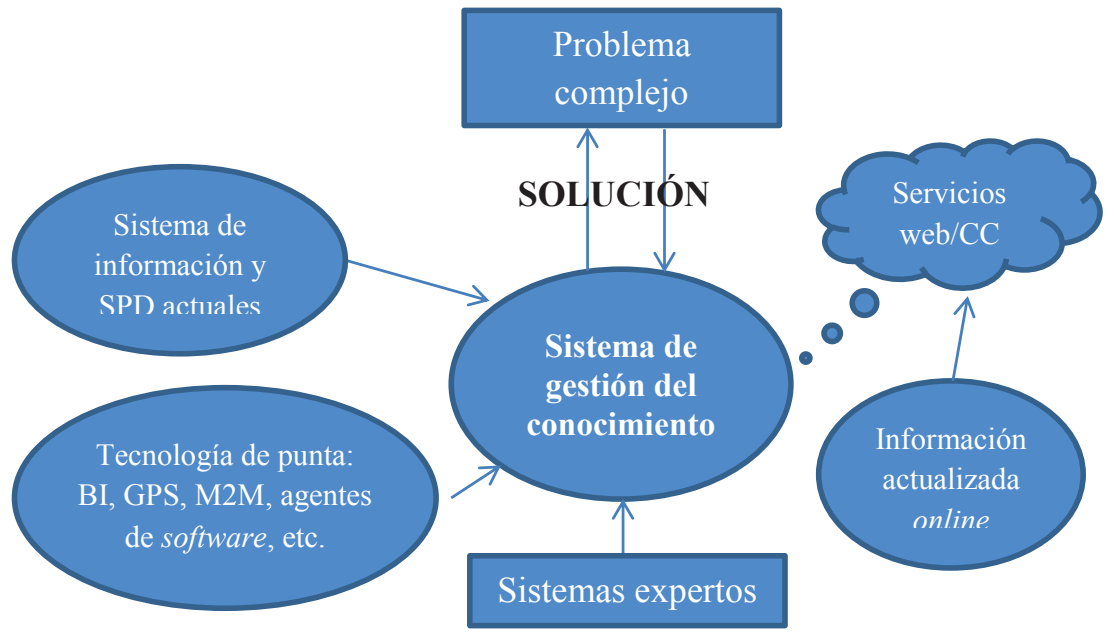

Elaboración propia.

\section{Conclusiones}

a) Es posible entender la gestión del conocimiento desde diferentes perspectivas empresariales, y a partir del análisis de problemas complejos se ha logrado desarrollar un modelo para un sistema de gestión del conocimiento basado en las tendencias más representativas de la gestión del conocimiento.

b) Entre estas perspectivas, la gestión del conocimiento se puede distinguir de seis maneras:

i. La estructura a partir de datos, información y conocimiento.

ii. Conocimiento alrededor del estado de la mente para el aprendizaje individual.

iii. Conocimiento necesario para un objeto que requiere construir activos para gestionar el conocimiento.

iv. Conocimiento alrededor de un proceso que debe gestionar el flujo desde la creación del conocimiento hasta su uso.

v. Conocimiento a partir del acceso a información de forma organizada y la recuperación del contenido.

vi. Conocimiento que se puede obtener a partir de la generación de capacidades que mejora la creación de competencias fundamentales.

c) Se presenta el modelo de generación de conocimiento como un momento dado en el tiempo y todo lo que hay antes y después se convierte en 
información. Luego se presenta el diseño de un modelo que permite gestionar los procesos de creación, almacenamiento, recuperación, transferencia y uso del conocimiento, donde se requiere de los sistemas heredados, y se utilizan tendencias en las tecnologías como agentes inteligentes, análisis de datos, tecnologías GPS, tecnologías M2M. Al mismo tiempo, se requiere de una nueva base en la experiencia acumulada con datos actualizados en línea con formas complejas de capturar información desde diferentes bases de datos en cualquier lugar del mundo.

\section{Referencias}

Alavi, M., \& Leidner, D. (2001). Knowledge management and knowledge management systems: conceptual foundations and research issues. MIS Quarterly, 1(25), 107-136.

Bernuy, A. (2005). Estrategias para el aprendizaje colaborativo y la transferencia efectiva del conocimiento. Ponencia presentada en el III Congreso Internacional de Científicos Peruanos. Universidad Nacional Agraria La Molina, realizado del 27 al 30 de agosto de 2005. Recuperado de http://eventos. spc.org.pe/cicp2005/papers/0012/Paper\%201/\%20AugustoBernuy\%20 rmcpperu2005\%20-\%20Estrategias\%20Apendiza.pdf

German Research Center for Artificial Intelligence. (s. f.). http://www.dfki.de/web/ research $/ \mathrm{km}$.

IC3K. (s.f.). http://www.kmis.ic3k.org/

Itainnova (3 de octubre de 2014). El proyecto europeo KM2.0 dispone de acciones formativas gratuitas sobre gestión del conocimiento para pymes. Recuperado de http://itainnova.es/proyectos/el-proyecto-europeokm2-0-dispone-de-acciones-formativas-gratuitas-sobre-gestion-delconocimiento-para-pymes

Massachusetts Institute of Technology - MIT Center for Collective Intelligence. (s. f.). http://cci.mit.edu/about_mitcenter.html

Michán, M. (18 de enero de 2011). Las acciones de Apple caen un 9.7\% en Europa tras la noticia de la retirada temporal de Steve Jobs. [Web log post]. Recuperado de http://www.applesfera.com/apple/las-acciones-de-apple-caen-un-97en-europa-tras-la-noticia-de-la-retirada-temporal-de-steve-jobs

Paniagua, E. (Coord.). (2007). La gestión tecnológica del conocimiento. Murcia, España: Universidad de Murcia/Editum. 
Reuters (10 de junio de 2011). Acciones de Apple bajan cerca de 1\% tras fallecimiento de Steve Jobs. Recuperado de http://www.americaeconomia. com/economia-mercados/finanzas/acciones-de-apple-bajan-cerca-de-1tras-fallecimiento-de-steve-jobs

Saiz, J. \& Olalla, B. (2010). Gestión del conocimiento y sistemas de calidad en los clusters de empresas familiares. Revista EAN, 68, 70-85.

Salinas, J., De Benito, B., Marín, V., Moreno, J., \& Morales, M. (2010). Herramientas y sistemas de gestión del conocimiento para el desarrollo de metodologías centradas en la colaboración y el intercambio. Ponencia presentada en el XIII Congreso Internacional Edutec 2010: E-Learning 2.0: Enseñar y Aprender en la Sociedad del Conocimiento. Bilbao, realizado del 3 al 5 de noviembre de 2010. Recuperado de http://gte.uib.es/pape/gte/sites/gte.uib.es.pape. gte/files/Herramientas\%20y\%20sistemas\%20de\%20gestion\%20del\%20 conocimiento\%20para\%20el\%20desarrollo\%20de\%20metodologias.pdf

Siemens. (2014). Industria 4.0, La cuarta revolución industrial. Recuperado de https://www.swe.siemens.com/spain/web/es/home/sostenibilidad/ innovacion/Pages/industria_4.0.aspx

University of London. (2012). Knowledge management. Recuperado de http://www. londoninternational.ac.uk/sites/default/files/knowledge-managementsample-study-guide.pdf 